\title{
A TIGHT AMORTIZED BOUND FOR PATH REVERSAL
}

\section{David GINAT}

Department of Computer Science, University of Maryland, College Park, MD 20742, U.S.A

\section{Daniel D. SLEATOR *}

Department of Computer Science, Carnegie-Mellon University, Pittsburgh, PA 15213, U.S.A.

\author{
Robert E. TARJAN ** \\ Department of Computer Science, Princeton University, Princeton, NJ 08544, U.S.A. and AT\&T Bell Laboratories, Murray Hill, \\ NJ 07974, U.S.A.
}

Communicated by $\mathrm{T}$. Lengauer

Received 28 July 1988

Revised 5 December 1988

Path reversal is a form of path compression used in a disjoint set union algorithm and a mutual exclusion algorithm. We derive a tight upper bound on the amortized cost of path reversal.

Keywords: Analysis of algorithms, path compression, disjoint set union, data structures, amortized efficiency

Let $T$ be a rooted tree. A path reversal at a node $\boldsymbol{x}$ in $T$ is performed by traversing the path from $x$ to the tree root $r$ and making $x$ the parent of each node on the path other than $x$. Thus $x$ becomes the new tree root. (See Fig. 1). The cost of the reversal is the number of edges on the path reversed. Path reversal is a variant of the standard path compression algorithm for maintaining disjoint sets under union [5]. It has also been used in a novel mutual exclusion algorithm $[2,6]$.

Suppose that a sequence of $m$ reversals is performed on an arbitrary initial $n$-node tree. What is the total cost of the sequence? Let $T(n, m)$ be the

* Research partially supported by DARPA, ARPA order 4976, amendment 19, monitored by the Air Force Aeronautics Laboratory under Contract No. F33615-87-C1499, by the National Science Foundation under Grant No. CCR 8658139, and by AT\&T Bell Laboratories.

* Research partially supported by NSF Grant No. DCR8605962 and ONR Contract No. N00014-87-K-0467. worst-case cost of such a sequence, and let $A(n, m)=T(n, m) / m$. We are most interested in the value of $A(n, m)$ for fixed $n$ as $m$ grows. As discussed by Tarjan and Van Leeuwen [5], binomial irees provide a class of examples showing that $A(n, m) \geqslant\lfloor\lim n\rfloor^{1}$, and their rather com-

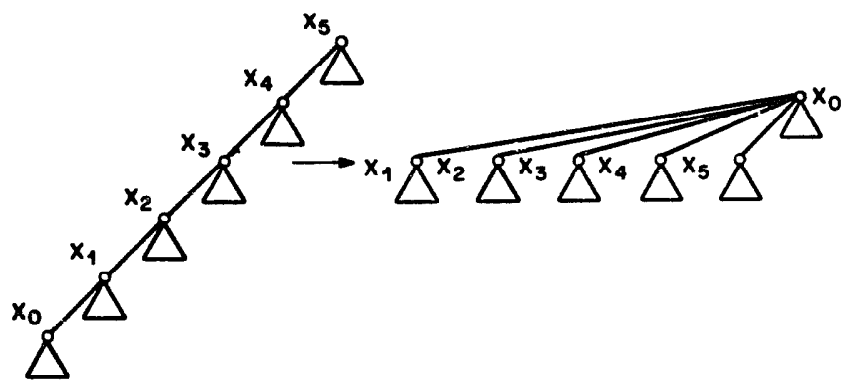

Fig. 1. Path reversal (triangles denote subtrees).

1 All logarithms in this paper are base 2. 
plicated and their rather complicated analysis gives an upper bound of

$A(n, m)=\mathrm{O}\left(\log n+\frac{n \log n}{m}\right)$.

Ginat and Shankar [2] prove that

$A(n, m) \leqslant 2 \log n+\frac{n \log n}{m}$.

We, shall prove that

$A(n, m) \leqslant \log n+\frac{n \log n}{2 m}$.

In the special case that the initial tree consists of a root with $n-1$ children, which is the case in the mutual exclusion algorithm, the bound is

$A(n, m) \leqslant \log n$.

To obtain the bound, we apply the potential function method of amortized analysis (see [4]). Let the size $s(x)$ of a node $x$ in $T$ be the number of descendants of $x$, including $x$ itself. Let the potential of $T$ be

$\Phi(T)=\frac{1}{2} \sum_{x \in T} \log s(x)$.

Define the amortized cost of a path reversal over a path of $k$ edges to be $k-\Phi(T)+\Phi\left(T^{\prime}\right)$, where $T$ and $T^{\prime}$ are the trees before and after the reversal, respect -ely. For any sequence of $m$ reversals, we have

$$
\sum_{i=1}^{m} a_{i}=\sum_{i=1}^{m}\left(i_{i}-\Phi_{i-1}+\Phi_{i}\right)=\sum_{i=1}^{m} t_{i}-\Phi_{0}+\Phi_{m},
$$

where $a_{i}, t_{i}$, and $\Phi_{i}$ are the amortized cost of the $i$ th reversal, the actual cost of the $i$ th reversal, and the potential after the $i$ th reversal respectively, and $\Phi_{0}$ is the potential of the initial tree. Since $\Phi_{0} \leqslant \frac{1}{2} n \log n$ and $\Phi_{m} \geqslant \frac{1}{2} \log n$, this inequality yields

$$
\sum_{i=1}^{m} t_{i} \leqslant \sum_{i=1}^{m} a_{i}+\frac{1}{2}(n--1) \log n,
$$

which in turn implies

$$
A(n, m) \leqslant \frac{1}{m} \sum_{i=1}^{m} a_{i}+\frac{n \log n}{\overline{2} m} \text {. }
$$

We shall prove that the amortized cost of any reversal is at most $\log n$, thereby showing that

$A(n, m) \leqslant \operatorname{lơ} \tilde{\Xi} n+\frac{n \log n}{2 m}$.

When the initial tree consists of a root with $n-1$ children, the bound drops to $A(n, m) \leqslant \log n$, since then $\Phi_{0} \leqslant \Phi_{m}$, and the extra additive term drops out.

Let $x_{0}, x_{1}, x_{2}, \ldots, x_{k}$ be a path that is reversed, and let $A$ be the amortized cost of the reversal. For $0 \leqslant i \leqslant k$, let $s_{i}$ be the size of $x_{i}$ before the reversal. The size of $x_{0}$ after the reversal is $s_{k}$ and the size of $x_{i}$ after the reversal, for $1 \leqslant i \leqslant k$, is $s_{i}-s_{i-1}$. We can thus write $A$ as

$$
\begin{aligned}
A= & k-\sum_{i=0}^{k} \frac{1}{2} \log s_{i}+\frac{1}{2} \log s_{k} \\
& +\sum_{i=1}^{k} \frac{1}{2} \log \left(s_{i}-s_{i-1}\right) \\
= & k+\frac{1}{2} \sum_{i=0}^{k-1}\left(\log \left(s_{i+1}-s_{i}\right)-\log s_{i}\right) \\
= & k+\frac{1}{2} \sum_{i=0}^{k-1} \log \left(\left(s_{i+1}-s_{i}\right) / s_{i}\right) .
\end{aligned}
$$

For $0 \leqslant i \leqslant k-1$, let $\alpha_{i}=s_{i+1} / s_{i}$. Note that $\left(s_{i+1}-s_{i}\right) / s_{i}=\alpha_{i}-1$. We have

$$
\begin{aligned}
A & =k+\frac{1}{2} \sum_{i=0}^{k-1} \log \left(\alpha_{i}-1\right) \\
& =\sum_{i=0}^{k-1}\left(1+\frac{1}{2} \log \left(\alpha_{i}-1\right)\right) .
\end{aligned}
$$

We now make use of the following inequality, which will be verified below: for all $\alpha>1,1$ $+\frac{1}{2} \log (\alpha-1) \leqslant \log \alpha$. From this inequality we obtain

$$
\begin{aligned}
& A \leqslant \sum_{i=0}^{k-1} \log \alpha_{i} \\
& =\sum_{i=0}^{k-1} \log \left(s_{i+1} / s_{i}\right)=\sum_{i=0}^{k-1}\left(\log s_{i+1}-\log s_{i}\right) \\
& =\log s_{k}-\log s_{0} \\
& \leqslant \log n \text {, } \\
& \text { since } s_{k}=n \text { and } s_{0} \geqslant 1 \text {. }
\end{aligned}
$$


This completes the amortized analysis. We verify the needed inequality by the following chain of reasoning:

$$
\begin{aligned}
0 & \leqslant(\alpha-2)^{2} \\
& \Rightarrow 0 \leqslant \alpha^{2}-4 \alpha+4 \\
& \Rightarrow 4(\alpha-1) \leqslant \alpha^{2} \\
& \Rightarrow \log (4(\alpha-1)) \leqslant \log \left(\alpha^{2}\right) \\
& \Rightarrow 2+\log (\alpha-1) \leqslant 2 \log \alpha \\
& \Rightarrow 1+\frac{1}{2} \log (\alpha-1) \leqslant \log \alpha .
\end{aligned}
$$

We conclude some remarks. The definition of the potential function used here has been borrowed from Sleator and Tarjan's analysis of splay trees [3]; it has also been used to analyze pairing heaps [1]. As in the case of splay trees, the upper bound can be generalized in the following way. Assign to each tree node $x$ a fixed but arbitrary positive weight $w(x)$. Define the total weight of $x$, $t w(x)$, to be the sum of the weights of all descendants of $x$, including $x$ itself. Define the potential of the tree $T$ to be

$$
\Phi(T)=\frac{1}{2} \sum_{x \in T} \log t w(x) .
$$

A straightforward extension of the above analysis shows that the total cost of a sequence of $m$ reversals is at most

$$
\sum_{i=1}^{m} \log \left(W / w_{i}\right)+\Phi_{0}-\Phi_{m},
$$

where $w_{i}$ is the weight of the node $x_{i}$ at which the $t$ th reversal starts and $W$ is the sum of all the node weights.

Choosing $w(x)=1$ for all $x \in T$ gives our original result. Choosing $w(x)=f(x)+1$, where $f(x)$ is the number of times a reversal begins at $x$, gives an upper bound for the total time of all reversals of

$$
\sum_{i=1}^{m} \log \left(\frac{n+m}{f\left(x_{i}\right)}\right)+\frac{1}{2} \sum_{x \in T} \log \left(\frac{n+m}{f(x)}\right) .
$$

It is striking that the "sum of logarithms" potential function serves to analyze three different data structures. We are at a loss to explain this phenomenon; whereas there is a clear connection between splay trees and pairing heaps (see [1]), no such connection between trees with path reversal and the other two data structures is apparent. In the case of path reversal, the sum of logarithms potential function gives a bound that is exact to within an additive term depending only on the initial and final trees. It would be extremely interesting and useful to have a systematic method for deriving appropriate potential functions. The three examples of splaying, pairing, and reversal offer a setting in which to search for such a method.

\section{Acknowledgment}

The first author thanks D. Mount and A.U. Shankar for valuable discussions and useful comments.

\section{References}

[1] M.L. Fredman, R. Sedgewick, D.D. Sleator, and R.E. Tarjan, The pairing heap: a new form of self-adjusting heap, Algorithmica 1 (1986) 111-129.

[2] D. Ginat and A. Udaya Shankar, Correctness proof and amortization analysis of a $\log N$ distributed mutual exclusion algorithm, Tech. Rept. CS-TR-2038, Department of Computer Science, University of Maryland, 1988.

[3] D.D. Sleator and R.E. Tarjan, Self-adjusting binary search trees, J. Assoc. Comput. Mach. 32 (1985) 652-686.

[4] R.E. Tarjan, Amortized computational complexity, SIAM J. Alg. Disc. Meth. 6 (1985) 306-318.

[5] R.E. Tarjan and J. van Leeuwen, Worst-case analysis of set union algorithms, J. Assoc. Comput. Mach. 31 (1984) 245-281.

[6] $M$. Trehel and $M$. Naimi, A distributed algorithm for mutual exclusion based on data structures and fault tolerance, In: Proc. Sixth Ann. Internat. Phoenix Conf. on Computers and Communication, Scottsdale, AZ (February 1987) 35-39. 